\title{
Safety Management of Small-Sized Chemical Waste Treatement Facilities in Victoria, Australia
}

\author{
Manikam Pillay
}

VIOSH, School of Health Sciences, University of Ballarat, Ballarat, Australia

\begin{abstract}
With the increased use of chemicals in industry, facilities for treating chemical wastes are becoming an important part of the modern society. In Australia, chemical waste treatment facilities are largely small - sized businesses, employing less than twenty people. Because of their small size these facilities have generally escaped the attention of regulators, researchers and policy advisors. This is a significant concern because many of these facilities may continue to operate with substandard safety practices and, if left unchecked, these facilities are prime candidates for organizational disasters and major accidents.This paper discusses the findings of a pilot study investigation undertaken to assess safety management practices across a sample of small-sized chemical waste treatment facilities in the state of Victoria, Australia. Results of this study provide useful information to waste, environment and health and safety advocacy groups, researchers, emp loyees and employers interested in prevention of chemical related incidents, and to government agencies involved in the regulatory administration of safety.
\end{abstract}

Keywords Chemical Waste Treatment Facilities, Safety MAP, Small Business

\section{Introduction}

Chemical waste treatment facilities (CWTFs) have become an important part of the modern society because of the relatively high reliance of chemicals. According to the Australian Bureau of Statistics (ABS) estimates there are about 2667 such facilities employing over 26000 employees directly, with over 5900 in other associated industries[1]. Most of these are small businesses employing less than ten people involved in a range of activities such as transportation, recovery or recycling, treat ment and disposal. Due to the hazardous nature of products they deal with CWTFs not only pose public health risks but are also prime candidates for chemical - related fires and explosions.

In order to manage such risks a range of legislative controls have been implemented. In Victoria, Australia, these include the Occupational Health and Safety Act 2004 (OHSA), the Dangerous Goods Act 1985 (DGA), the Dangerous Goods (Storage and Handling) Regulations 2000 (DGSHR) and the Occupational Health and Safety Regulations 2007 (OHSR). OHSA requires chemical waste treatment facilities (CWTFs) to provide and maintain a safe working environ ment, safe plant and systems of work, make arrangements for safe use, handling, storage and transport of substances, maintain workplaces and welfare facilities,

* Corresponding author:

pillay.manikam@gmail.com (Manikam Pillay)

Published online at http://journal.sapub.org/mm

Copyright (C 2012 Scientific \& Academic Publishing. All Rights Reserved and provide information, instruction, training and supervision for employees[2]. DGA and DGSHR 2000 require CWTFs to notify the safety regulator if they stored or handled dangerous goods above specified threshold quantities, and of any chemical related incidents such as spills, explosions and/or fires[3]. So me CWTFs may also be subjected to major hazard facility (MHF) requirements if quantities of chemicals they dealt with exceeded specified thresholds[4]. Such CWTFs fall under the state's safety case regime and are required to identify all hazards likely to cause a major incident, conduct safety assessments, adopt control measures, and develop and imp lement a safety case, a safety management system and emergency plans [5].

The safety legislation is performance based and modelled on the self-regulation in line with that suggested by Roben's[6]and adopted by most European countries. This approach gives industry the opportunity to establish its own standards, benchmarks and enables organisations to adopt management systems. Hence, irrespective of the size of an organisation, the legal requirements of safety are the same, so developing, implementing and maintaining a safe system of work, managing safety risks, consulting and those dealing with documentation and training are identical and enforceable to the same extent. However the small-sized nature of their businesses means most CWTFs generally escape the attention of safety regulators, policy makers, and researchers, with many continuing to operate with poor and/or substandard safety practices. Limited efforts have been directed at examining the safety performance and the effectiveness of safety management at these facilities. This 
paper, based on a pilot study in South-Eastern Victoria, Australia, is a first attempt at addressing this gap.

\section{Literature Review}

Monk[7] examined the contributions of safety manageme nt systems to reductions in injury and disease at construction sites through an audit tool developed by the Construction Industry Development Agency (CIDA). Performance measures in this study were based on workers compensation claims and included cost of claims; cost of claims per emp loyee; average lost time rate; and injury frequency rates. 14 management system elements were also assessed using a Likert-scale of zero (non-existent) to five (sustainable best practice). Monk's research showed large differences between health and safety performance of small contractors (10-19 employees) when compared to larger companies (>150 employees). She concluded that, based on the assessment of the 14 elements, the small contractors generally did not meet legal requirements[7].

Whilst the study could be criticised for relying the on use of lag indicators such as workers compensation and injury frequency rates, the main strength of this study lies in combining these with safety management elements in determining OHS performance. Many CWTFs invariable adopt safety management systems, and these could be used for assessing their performance alongside lag indicators.

Poulsen, et al.[8] and[9] conducted comprehensive reviews of occupational exposure and health problems associated with waste collection, sorting and recycling using the Danish Registry of Occupational Accidents and Diseases data from 1984 to 1992. High incidence rates of gastrointestinal problems, irritation of the eye and skin, as well as organic dust toxic syndrome (influenza-like symptoms cough, muscle pains, fever, fatigue, and headache) were reported to be among workers collecting the biodegradable fraction of domestic waste. The few data available on exposure to bio-aerosols and volatile compounds indicated the waste collectors were simultaneously exposed to multiple agents such as dust containing bacteria, endotoxin, mould spores, glucans, volatile organic compounds, and diesel exhaust. Higher levels of pulmonary and gastrointesti nal problems were reported from waste recycling facilities; these were associated with higher exposures to airborne dust containing micro-organisms and microbial agents. Noise levels in excess of $90 \mathrm{~dB}(\mathrm{~A})$ were also reported from recycling plants. The authors concluded that larger-scale research including surveys and analytical epidemiologic studies were needed to identify the actual causes of these occupational diseases $[8,9]$.

Such long-studies can be undertaken as part of doctoral research studies; however, it is largely dependent upon the effectiveness of systems that are availab le capturing data on occupational diseases and workplace exposures.

Riley and Weyman[10] surveyed permit-to-work (PTW) to develop practical industry guidance on how effective PTW systems could be designed and implemented. 55 semi-structured interviews across 19 small and medium-sized plants operating in the UK onshore chemical industry were conducted, and completed PTWs were also examined to determine whether important system functions were captured. The research revealed lack of hazard identification during permit initiation, with only $20 \%$ of companies having this function on the permit[10]. Where these were done they were usually done mentally 'in the authoriser's head' and relied entirely on the knowledge and experience of the authoriser to ask enough 'what if?' type of questions, and half of the companies had 'copied' their permit system and permits with or without any changes from other sources hence were not site specific[10]. This was often attributed to parent company imposing the system, which were generally developed in a "top down" manner, with little or no consultation with users[10].

This was largely an evaluative study, and the recommend ations arising out of this study providing practical guidance on how PTWs could be effectively designed and used in small and mediums sized enterprises.

Fairfax[11]reviewed safety and health plans of eighteen Superfund remediation contractors as part of ten comprehen sive site audits conducted jointly by Occupational Health and Safety Administration (OSHA) and Environmental Protection Authority (EPA) officers. Common deficiencies identified at the sites included absence of written health and safety plans, inadequate engineering controls, work practices, personal protective clothing, site monitoring and emergency responses[11].

This study utilised a combination of site inspections, data analysis, sampling, and auditing methods, and involved a cross-section of workplace personnel, allowing for greater stakeholder engagement at the site level. This was a more comprehensive study involving multiple data collection approaches at different layers of organisations. One main drawback of this research strategy, however, is the involvement of two government agencies to assess compliance; this can not only be very time-consuming but also can place additional strains on small businesses. The auditing methodology can however, be a useful way of profiling safety performance at any point in time.

Gladding and Coggins[12] evaluated air quality and health effects of organic dusts from household waste recycling in two Materials Recovery Facilities (MRFs) in the UK. Data was collected on 'cleanliness' of the waste, micro-organisms, lung-function capacity of operators', and nature of organic dusts. Results from this study showed that, in many categories the control group displayed higher degrees of symptoms, such as throat and eye irritation, headache and joint pains. Many of the workers in the experimental group were quite certain that their symptoms were related to work, apart from a small percentage with cough and phlegm and dry cough (thought due to smoking) and tiredness and joint pains. The authors concluded that longitudinal studies were needed to monitor workers before they started and during the course of their emp loyment[12].

This research may provide support claims that CWTFs, 
especially those which are involved in the storage, handling and processing of biological, physical and chemical wastes, are likely to expose their workers to a range of hazards, some of which may result in illnesses. This study could be reproduced in the Australian context as part of a larger research aimed at examining specific occupational health and hygiene hazards and risks that workers in CWTFs are most likely to be exposed to.

Lamm[13]examined occupational health and safety (OHS) practices, and the role that small accounting practices played in promulgating safety practices in New Zealand small businesses. Self-administered questionnaires were administered to 140 chartered accountants from the Auckland Chartered Small Practice Group with a response rate of $27 \%$. This research demonstrated that small accounting firms frequently acted as 'intermediary advisors' between the $\mathrm{OH} \& \mathrm{~S}$ regulatory agencies and the small business sector. The authors attributed this to the fact that there was little direct contact between the OHS agency, and this was likely to influence the way in which regulatory agencies established OHS compliance in s mall workplaces [13]. Lamm questioned the level of expertise accountants had in the area of OH\&S compliance and practice and their ability to provide an extended compliance service outside their traditional area of accounting and finance, suggesting that there was a possibility that the small business sector circumvented the OHS government agencies when providing compliance assistance[13]. He further argued the proposition that small business employers used their accountant for advice on the OH\&S law highlighted the complex and informal OHS compliance patterns that occurred in the small business sector[13].

Lamm's study reinforces that OHS policies and practices derived solely on the experiences of larger organisation were necessarily transportable to small business sector. From a research point of view this is suggestive that any tools used for assessing safety in larger organisations may need to be adapted for use in smaller business.

McVitte, Banikin and Brocklebank[14]assessed the influence firm size had on lost-time injury rates. As part of this study the researchers reviewed records from the Workers' Compensation Board of Ontario relating to injuries, man-hours, payroll and firm size. The results showed that over a five-year period injury frequency increased consistently as firm size decreased. Large firms had a consistently lower frequency of Lost Time Injuries compared to s maller firms[14]. The authors concluded that such trends were evident over a number of years and appeared to be unaffected by cyclical employ ment patterns. The authors argued that (i) public policy issues relating to OHS in the construction industry needed to be focussed more on small to medium sized firms instead of large firms; (ii) new regulations and standards designed for larger employers or which were capable of readily implemented by large firms may not yield the type of improvement sought, since the injury frequency within that group was already low; and (iii) newer approaches to enforcement, education and dissemination information dissemination specifically targeted to the needs of smaller emp loyers were needed[14]. This study provided support to a view that smaller firms are perhaps more hazardous and dangerous than larger ones.

Eakin and MacEahen[15]undertook a qualitative studyto investigate health and social relations in Canadian small enterprises in order to develop a theory on the social production of illness and injury in the workplace. Data was collected from 40 semi-structured interviews. The researchers found that personalised nature of relationships and the low polarisation of employer-employee interests shaped workers' perception of employment relationships and health at the workplace[15]. In addition, strained authority relationships at work formed a key social context in which health and injury was constructed, with bodily experiences problematized where there were conflicts between workers and their supervisors. The researchers concluded that unheeded illness claims deepened feelings of distrusts and blame, caused deteriorations in labour relations and re-produced social conditions of illnesses[15].

In Australia there have been concerted efforts taken by both the Federal and state governments to ensure all workpl aces, irrespective of their size and nature of operation, have mechanisms in place that encourage participation and consultation between supervisors, managers and workers. In CWTFs avenues for this to occur are during induction training, during risk-assessments of chemicals, and when there are decisions being made about risk control measures. Any assessment of safety performance in this sub-sector may need to include this social aspect of safety management.

Hale et. al.[16]investigated the management of safety in maintenance activities in the chemical process industry in the Netherlands. A theoretical model of an ideal maintenance management system incorporating safety was established and tested through peer reviews in five companies in different industries which had showed higher safety risks and requirements and had good reputations for ma intenance management, which was then used to carry out a secondary analysis of available data on maintenance accidents[16]. In addition, an audit checklist derived from the model was utilised to conduct an in-depth assessment of the management systems of eight major hazard plants[15]. Finally, the model and audit were used to construct a questionnaire, which was sent to 82 major hazard companies, and the triangulated data analysed to identify opportunities for improvements in the maintenance management systems[16]. The research identified that the main shortfalls were evident in the support provided to middle levels of management in translating safety policy into an effective maintenance concept and into planning, procedures and resource management which gave lower attention to safety[16].

As an in-depth study of safety management in MHFs, this research points towards some issues that need to be included in any assessment tool aimed at developing an 
understanding of the safety performance of CWTFs. One is the policy, planning and resourcing of safety by managers and operators of CWTFs. Another is the effectiveness of systems and approach used for maintenance of plant and equipment used in CWTFs.

Vassie and Cox[17]conducted a preliminary study to examine the interests for a voluntary health and safety management system certification scheme amongst small and medium-sized enterprises in United Kingdom through postal semi-structured questionnaires. A mix of multiple choice and open questions covering the nature of the SME's business activities and respondents' views on participation in voluntary management accreditation schemes were sought from 625 SMEs spread across UK, in combination with three focus groups comprising twelve SME managers from engineering and manufacturing companies[17]. The authors concluded that the main reasons for any interest in a such certification schemes included the desire to imp rove or ensure the health and safety of employees, and to raise awareness across the industry. The main features required in any such schemes were that they be of low cost, easy to maintain and tailored to suit the needs of the sector the organisations operated within. The researchers also found that barriers perceived to most likely limit participation included bureaucracy associated with the scheme following small businesses' experiences with quality systems, the resource implications and the importance of health and safety to the business. The authors concluded that a voluntary certification scheme could be a usefulway of recognising safety and improvements in UK SMEs, but this concept needed to be tested with a larger sample size[17].

An, Englehardt, Fleming and Bean[18]examined safety performance of municipal solid waste workers (MSW) in Florida using data from the Florida Workers' Compensation scheme for a five year period. The researchers identified that the MSW workers were more likely to be exposed to musculoskeletal and dermal injury risks such as strains or sprains, contusions, fractures, and lacerations. Waste collectors (drivers or helpers) had a higher risk of injury than other workers in the MSW industry. Whilst injury rates for all Florida industrial workers decreased, the injury rates of MSW workers almost doubled from 1993 to 1997. It was noted that workers' compensation claims represented a small fraction of total injuries. On the basis of reported and studied occupational diseases, and workers' high exposure to microorganisms and dust, occupational illness rates were considered to be high amongst MSW workers. The researchers concluded that further studies on different waste management worker subpopulations were needed to provide detailed information to reduce these risks.

Harrison, Woodhouse and Dowson[19]conducted occupa tional health audits at 36 National Health Service (NHS) trusts located in the Northern and Yorkshire regions of England using a self-administered questionnaire (SAQ) they derived from a series of eight standards established by the NHS, which was distributed to the occupational health departments of the trusts and completed by the senior nurse. The sites were also audited by a qualified and experienced occupational health nurse using a similar questionnaire, with a number of additional questions aimed at verifying the information obtained from the SAQ. Further supporting information was obtained through interviews with other key informants, supporting data collected through documents such as policies, procedures; training programmes and occupational health notes and records. The SAQ was designed so that a 'yes' or 'no' answer could be chosen for most of the questions[19].The research showed a considerable range of performance in health and safety, pre-employment, assessment, infection control, health surveillance and health promotion, which the authors argued had implications for both the safety of the working environment and the fitness of staff performing the work[19]. Most research sites had a safety policy approved at board level, and well-defined delegated responsibilities for the managing health and safety, which the authors suggested was a good starting point; however, a more proactive approach to the identification of workplace hazards and associated risks, followed by imp lementation of policies to control those risks were required[19]. Some hazards such as manual handling and fire were well assessed and managed, but other hazards such as hazardous substances, violence to staff, stress at work and noise were not as well identified, assessed or managed[19].

This was a very comprehensive study in which data was collected over a six-month period using a mixed-methods approach. The approach can be used for an in-depth, doctoral level research project; however, theuse of a YES/NO response could be useful way of conducting a pilot study of safety performance of CWTFs. In addition, the types of data collected in this study, including training plans, organisational policies, and similar documents are generally required to be maintained under Victoria's safety laws, and they can form part of the data collection strategy.

Vassie, Tomàs and Oliver[20]surveyed SMEs in Spain and UK to establish their approach to health and safety management and gain their views on participating in voluntary management accreditation schemes. Data was collected through a self-administered questionnaires posted to over 900 SMEs with response rates of $11.4 \%$ (UK) and $13.9 \%$ (Spain). The researchers found that there were some key differences between UK and Spanish SMEs. There was (a) an enhanced level of awareness of health and safety legislation; (b) a higher prevalence of safety and quality management systems, and (c) greater involvement of senior managers in managing health and safety in UK enterprises[20]. Interest was expressed in a voluntary management accreditation scheme for health and safety by over half the UK and Spanish sample. Furthermore, those enterprises participating in a voluntary quality management accreditation scheme were more likely to be interested in a voluntary scheme for health and safety management. The authors concluded that the results were based on a small 
sample size and more biased towards the manufacturing sector, and that further work was required to test the concept among a wider industry group[20].

Some of the elements explored in this study, such as manager's awareness and their involvement in safety are part of the basic guidance material issued by safety regulators. So any assessment of CWTFs can include making an assessment of this aspect of safety management in organisations.

Bull, Riise and Moen[21]investigated whether any heath, environment and safety (HES) factors registered by organisations through visits at the start of a study could be used to predict the risk of injury in subsequent years. The researchers registered twelve HES factors (including injury awareness, programme for action, employee participation, training and use of personal protective devices(PPDs)) through the use of questionnaires and observations made during production in a sample of two hundred and eighty eight small mechanical enterprises[21]. Using regression analysis, the researchers concluded that increasing the use of PPDs and safety equip ment on machines was most likely to increase prevention of injuries, and that frequent inspections with feedback to workers was most effective means of reducing injuries[20].

The conclusions in this study point towards the need for both engineering and PPD as suitable control measures for preventing injuries in small businesses, and this aspect needs to be part of any assessment tool aimed at investigating safety performance in CWTFs.

Champoux and Brun[22]conducted an exploratory research to develop a picture of OHS management in Quebec small sized enterprises (SSEs)which employed less than fifty workers. Telephone interviews were conducted with 223 with small-sized enterprise owner managers across metal products manufacturing (103) and garments (120) sectors. Data collected included enterprise characteristics, workforce characteristics and organization of work, diagnosis of OHS its management. The researchers found that SSE owner-managers were isolated, over-worked, did not use the services offered by OHS sector associations, and generally were not formally organised into business groups. Most appeared to be poorly informed and many did not realize the extent of the OHS risks common in their operations. The results also showed that there were significant differences in OHS management among the SSEs. The authors concluded that interventions in SSE, including providing any support to OHS management, needed to be aimed at specific sub-groups of the SSEs. Moreover, in order to be effective, any external interventions needed to take into account the SSE practices, their owner-managers' perceptions and their management styles[22].This study lends some support to an argument that many small businesses are vulnerable because they do not have their own network by through which they can have a voice in the development of national level policies.

Larsson[23]surveyed 100 businesses in Melbourne from ten different industries as part of a small business safety survey. Face-to-face interviews were held with participants to gather information on a wide range of areas using perception analysis and cross-checking incident and claims data using the VWA database. The author concluded that there was no real proof that size of an establishment was an important factor in health and safety understanding and decision- making.

The inclusion of 100 participants allowed for good representation of candidates, and gave a more reliable data set. However, there are significant time constraints in interviewing 100 candidates. Of greater significance is the practice of data-matching claims history with information collected from the businesses. This method of enquiry can be useful in verifying information collected through audits.

In another study Tischer and Scholaen[24]evaluated how a three-step guide for safe management of chemicals were implemented in Indonesian SMEs. The research sites included five companies, three of which were involved with textiles, the fourth a tannery and the fifth a paint producer and drum recycler. Data was collected through field visits lasting up to four hours, extensive discussion with the owners and a 'walk-through' to identify hot spots. The research identified that because most organisations did not have a material safety data sheet (MSDS), managers were not aware of the hazardous properties of the chemical products they were using. This meant that small companies were generally unable to conduct sophisticated risk assessment because they had insufficient infrastructure and the information necessary for the risk assessment process. Smaller companies also lacked systematic organizational procedures and documentation; there was a lack of priority and responsibility allocated for safe management of chemicals, and limited financial and human resources. These companies expressed an interest in simple criteria for identifying hot spots and rules of thumb to reduce losses of chemical materials. They needed to see what benefits could be achieved with the guide in a short time-frame for them to be convinced that putting efforts into such an activity was worthwhile. The research found that the medium-sized and larger companies did have more MSDSs on site; however, most did not understand the importance of having them and did not know how to use them. Moreover, most of the larger companies did provide personal protective equip ment (PPE) and some workers even use it. However, the design was often insufficient to offer adequate protection. In some instances, workers have made their own face masks or wrap towels around their face, but with very little understanding of potential health effects due to exposure to chemicals.

The approach used in this study can be adapted for use in Victoria. This is because MSDS are one of the main documents that are required to be maintained by companies that use, store and handle chemicals[3]. In addition, risks assessments and use of personal protective equipment are mandated in the leg islative controls for health and safety.

Walters and Lamm[25]explored the complex and difficult relationship between existing approaches for regulating the work environment and what it meant for small business. 
The authors note that there was clear international evidence of a widespread problem of health and safety performance in small businesses. The authors suggested this was not because such workplaces were inherently more hazardous than their larger counterparts but that the arrangements for workers' health, safety and well- being were underdevelope d. Some of the reasons for the underdevelopments included 'structures of vulnerability' in small businesses that effectively reduced the will and capacity of managers and workers to make effective arrangements for health and safety either jointly or separately. Hence the authors argued that it is senseless to introduce OHS regulations, policies and preventative initiatives unless the reasons why and how small business employers comply and their attitudes to their workers' health and safety were clearly understood.The authors further note that here was a plethora of initiatives for achieving better health and safety arrangements and performance in small businesses; however, what worked best was face-to-face contact with change agents connected to the health and safety system, such as OHS inspectors and practitioners.

Walker and Tait[26]assessed the effectiveness of an appro ach which enabled small enterprises set up and operate a simple health and safety management system (SMS), comprised of a health and safety policy, risk assessment and development of appropriate control measures. The effectiveness of the approach was assessed by follow up visits to 24 enterprises that had previously received help. The assessment utilised interview, inspection of premises, document examination, and assessment of training and maintenance standards. The researchers found that the strategy was effective in almost all the cases in helping enterprise managers to produce adequate policy statements and risk assessments, and to introduce an effective health and safety management system. However, the researchers found that many of the necessary arrangements were actually in place prior to the intervention. The authors argued that the intervention may have been effective in bringing these diverse arrangements together and in augmenting them in order to produce a more coherent management system. The researchers also found that, as a result of the intervention, the enterprise manager had developed the necessary knowledge and confidence to maintain and further improve the system that was in place.

This was largely an evaluative study of an initiative introduced by the health and safety regulators. Some of the elements that were explored in this study, such as health and safety policy, risk assessment and appropriateness of control measures, can be included as elements of any assessment tool. Other elements that were considered in this study, such as document associated with training and maintenance can also be included. And the research strategy used, i.e. interviews and inspection of premises, are normally part of a safety audit, and this could be used for assessing safety performance of CWTFs.

Statheropoulos, Agapiou and Pallis[27]examined volatile organic compounds (VOCs) that evolved from urban household waste disposal bins. As part of a case control study, the researchers examined waste of various loads (full, empty, partially filled bins), remained uncollected in the containers for variable time and under a range of weather conditions. VOCs were analysed using thermal desorption/ gas chromatography/mass spectrometry (TD/GC/MS) on over 150 compounds with the 30 most abundant quantified. When waste accumulated in bins under unforeseen circumstances, some compounds produced were likely to exceed olfactory and safety thresholds hence represented potential sources of adverse health impacts[27]. Benzene and dimethyl di-sulfide levels determined in this work were found around safety and odour threshold levels. The high levels of alkanes, alkyl-benzenes, and terpenes found are responsible for the undesirable odours. Duration of waste exposure, along with load and prevailing weather conditions were suggested to affect the evolution of VOCs and their concentration.The researchers concluded that monitoring of VOCs from organic waste material was a useful way of assessing health risk of exposure not only to waste handling personnel, but also the city population as well.

This research provided early indications of types and levels of VOCs produced in urban waste disposal bins. The authors concluded that larger scale measurements were required to establish guidelines concerning urban health risk ass essmet.

Tolvanen and Hänninen[28]assessed occupational health risks of a waste incineration plant. As part of this study the researchers looked at concentrations of microbes, endotoxins, and dust and noise levels in three different working areas of the plant. Microbe analyses were conducted. The authors concluded that concentrations of bio-aerosols and noise levels were high enough in the waste bunker to be occasionally harmful employees health, and that concentrations of endotoxins were also harmful to emp loyees in the combustion area, with the noise level there exceeded the Finnish threshold value of $85 \mathrm{~dB}(\mathrm{~A})$. The total number of microbes (viable+dead) was also in the crane room. The researchers provided a number of recommendati ons for reducing risks to employees, including use of ear protectors to reduce exposure to noise and a class P3 respirator to reduce exposure to microbes, endotoxins and dusts.

In another study Tolvanen and Hänninen[29]examined environmental and occupational hygiene factors of a newly constructed kitchen bio-waste treatment facility in Finland. Concentrations of dust, microbes, endotoxins and noise levels were measured at the pre-processing and crushing hall, the bioreactor hall and the drying hall, and interviews held with employees regarding any work-related health problems they experienced. Occupational hygiene issues of concern included increased concentrations of microbes and endotoxins during waste crushing and in the bioreactor hall. Because employees complained of symptoms such as dry cough and rash or itching appearing once or twice a month, the researchers recommended the use of $\mathrm{P} 3$ respirator masks 
during dusty working phases. The noise level in the drying hall exceeded the Finnish threshold value of $85 \mathrm{~dB}$ (A). Qualitatively harmful factors for the health of employees were similar to other closed waste treatment plants in Fin land. Based on the quantitative assessments the authors suggested that the newly constructed treatment plant was better compared to some of the Finnish dry waste treatment plants, and that mechanical sorting, which produced a dry waste fraction for combustion and a bio-waste fraction for anaerobic treatment, was better for emp loyees compared to combined aerobic treatment and dry waste treatment.

Sørensen and Bach[30]examined the extent of differences between small and large enterprises in relation to work environment hazards and work environment management systems and assessment. The researchers combined two the 'Danish Work Environment Cohort Study' (which contains self-reported data on physical, chemical and psychological environment) and the 'Surveillance of Health and Safety Activities in Enterprises' datasets to identify if the differences were significant. Interviews were also conducted in 10 companies. The researchers concluded that, within a representative sample of Danish workplaces (i) size was positively correlated with physical working conditions in private-independent enterprises, with larger enterprises having better working condition; (ii) size was negatively correlated with physical working conditions in private-part enterprises, with smaller enterprises having better working condition; (iii) size was positively correlated with the quality of $\mathrm{OHS}$ management systems and workplace assessment activities for all enterprises, with larger enterprises having OHS management systems that were of a higher quality; (iv)size was weakly correlated with psychosocial working conditions, with smaller enterprises having better psychosocial working conditions irrespective of the type of ownership; (v) size relations to work environment conditions were identical across most industry groups, with type of ownership being more important than industry group[30].This study supports the view that smaller workplaces experienced more hazards and risks compared to larger ones.

The following points can be summarised from this review. Smaller business is likely to expose their workers to a range of physical, chemical and psychological hazards with different degrees of risks. Studies from a number of countries suggest smaller businesses may experience greater difficulties in terms of safety performance and management. Also, smaller businesses lack the resources of larger ones in terms of time, good staff and finance and are unable to keep abreast of the changes to OHS legislation, and they are less likely to adopt safety management systems unless such requirements are placed on them by law. What is unknown is the current knowledge about the state of safety performance in the CWTF subsector. This research aims to address this gap. More specifically it aims to explore the question "how well is safety managed in CWTFs in Victoria?”

\section{Research Methodology}

This pilot study was undertaken at small-sized chemical waste treatment facilities that were licensed by Environmental protection Authority Victoria (EPA V).An internet search was conducted on the Victorian EPA website to obtain a listing of waste treatment facilities by location. Telephone calls were made to all companies located in the South-Eastern region to identify facilities which employed twenty or less people. From these nine organisations were selected fro mthose that were located within easy reach of the researcher's work location. In order to encourage participation, the facilities were advised that the assessments would provide them with an opportunity to establish their baseline level of compliance to existing safety laws and identify any hazards and risks without any direct cost to them. This is essentially a convenience sampling strategy [31]and the results should not be treated as being typical of other CWTFs in Victoria.

To guide the data collection process, a five element safety assessment system framework based on in SafetyMAP (4th Edition)[32] was developedthat enabled the collection of data on:

- Demographic information of the participating organisations

- How well the facility met a set of set of performance standards across the above 5 elements and 28 sub-elements of SafetyMAP.

\subsection{Data Collection}

Site visits, lasting between 1 and 3 days, were conducted across the nine CWTF over a period of four months. During the site visits structured and semi-structured interviews were held with operations managers, leading hands, employees, health and safety representatives and health and safety committee members. In addition, walk-through inspections were undertaken to observe how activities were conducted and how risk control measures were implemented and maintained. Documents such as licenses and certificates of competencies, registrations, and records of induction and other training, risk assessments, job safety analyses, permits and clearance forms, policies and work procedures were also reviewed. Information gathered from the site audits were marked with a YES / NO response on an audit checklist. Information regarding claims, incidents and injuries were matched against information maintained by an Incident Notification and Workplaces Databases maintained by the state safety regulator.

\section{Results and Discussion}

\subsection{Claims and Injuries}

Within the set of nine companies examined over the study period, a total of 10 claims were from three companies were lodged; 1174 workdays were lost from these incidents, and 
the cost of claims amounted to \$159 000. Based on these figures the following safety performance statistics can be derived:

- average cost of claims (cost of claims divided by number of claims) amounted to \$15 900; and

- average lost time rate (ALTR - number of days lost divided by number of claims) was 117.4.

These statistics can be compared, on a relative basis, to the performance of SMEs identified[31]. The researchers identified that SMEs generally had a and higher average costs per claim The authors attributed this to poorer 'return to - work' options, and lack of information on prevention and management. This study did not examine the way in which injured workers were managed in CWTFs.

\subsection{Health and Safety Hazards}

Health and safety hazards were identified through physical observation, review of work and maintenance practices, and re-examination of reported claims and incidents. The eights common hazards identified are shown in Table 1.

Table 1. Eight most common hazards identified (n=9)

\begin{tabular}{|c|c|c|}
\hline & Hazard & $\begin{array}{c}\% \text { of } \\
\text { workplaces }\end{array}$ \\
\hline 1 & $\begin{array}{c}\text { Interaction between pedestrians and mobile } \\
\text { plant because of inadequate traffic } \\
\text { management controls }\end{array}$ & 78 \\
\hline 2 & Inadequate maint enance of fire protection & 78 \\
\hline 3 & $\begin{array}{c}\text { out of date and/or emergency response plans } \\
\text { that were not fully implemented }\end{array}$ & 78 \\
\hline 4 & $\begin{array}{l}\text { absence of written maintenance and schedule } \\
\text { for plant }\end{array}$ & 56 \\
\hline 5 & $\begin{array}{c}\text { out of date and/or incomplete chemical } \\
\text { registers }\end{array}$ & 56 \\
\hline 6 & incompatible storage of chemicals & 56 \\
\hline 7 & $\begin{array}{l}\text { absence of written procedures and/or risk } \\
\text { assessments }\end{array}$ & 56 \\
\hline 8 & wet and slippery work platforms & 56 \\
\hline
\end{tabular}

Some of these hazards are also common to the findings of studies at thermal waste treatment plants by the Fairfax study[11]. These included, on a comparative basis:

- written health and safety plan (compares with absence of maintenance plan for plant and fire protection),

- work practices (compares with inadequate maintenance and inspections of fire protection systems, incomp lete / out of date register of chemicals, incompatible storage of chemicals, absence of written work procedures or instructions for operators, unlabelled chemicals in transfer and storage vessels, and inappropriate electrical equip ment or inadequate inspection and testing regime),

- site monitoring practices (compares with absence of maintenance plan for plant and fire protection), and

- emergency response programs (inadequate maintenance and inspections of fire protection systems, emergency response plan not current or implemented, and blocked emergency exits).

\subsection{Safety Management}

The results of safety management examined are presented under (i) management, planning and resourcing, (ii) consultation and employee engagement, (iii) employee training and development, (iv) risk and incident management, (v) emergency management and (vi) monitoring and review.

\subsubsection{Management Planning and Resourcing}

The aim here was to examine if the company had policies, procedures in place, whether they complied with EPA V registration and VWA notifications, had qualified or competent (or access) persons to advise them safety and / environmental requirements, whether the most senior management representative was aware of legal and compliance obligations, and if procedures and protocols were in place to deal with health and safety concerns. All organisations had adequate arrangements for management, planning and resourcing, although there were variations between the facilities. Only $44 \%$ had access to a qualified and/or competent safety profession. 78\% had established arrangements for resolving health and safety issues raised by employees, visitors and contractors. The arrangements included formal and informal measures (daily chats, supervis ors working with employees). 56\% of management representatives were aware of their legal obligations for safety, $44 \%$ of the sample of organisations met all the sub-elements examined. These four organisations also had established integrated management systems in place.

The findings support conclusions made by Walters and Lamm[23] that low management training and skills and lack of resources were among those factors affecting OHS in the small business sector.

\subsubsection{Consultation and Employee Engagement}

The aim was to gain an understanding of whether there were arrangements in place for managers to consult with employees on changes associated with introduction and/or revision of controls and in the risk management practices. $33 \%$ of the workplaces had elected designated workgroups (DWGs) as required by the state safety legislation, and arrangements for their participation. 44\% actually consulted with employees during identification of hazards and assessment of risks, safety committees, records of meetings and communication of issues discussed. $56 \%$ also consulted when implementing or revising controls. Only 22\% of the organisations met all the requirements of adequate consultation; again, these included companies which had established management systems.

The higher percentage of employee engagement when revising or implementing controls appears to be a unique in these facilities. The results contrast with earlier findings associated with decision-making in small business identified by Larsson, where only $32 \%$ of managers were likely to consult with staff. It has been widely acknowledged that consultation between employees and management produces good gains for any business, and is an adoption of 
self-regulation at workplace level. Involving employees, providing incentives, communicating and listening to them are suggested to be among the most effective methods of instilling safe behaviour.

The above results also correlate to the findings for management planning and resourcing. Difficulty in getting access to safety resources and understanding of legal requirements could be a hindrance to establishing more formal consultative structures at over $56 \%$ of the workp laces examined.

\subsubsection{Employee Train ing and Development}

The aim here was to assess whether employees received any induction, job-related and site specific emergency response training. Requirements for site inductions and emergency response training existed in $22 \%$ of companies, while industry specific training was provided by $33 \%$. Comparing the results with the findings of management planning and resources above, it is seen that while requirements for registrations (including mobile plant and delivery vehicles) were adequate in $89 \%$ of the companies, but only $44 \%$ had safety expertise or access to such expertise when required. This was generally through external consultants. This suggests that, while significant efforts are made by the employers to provide the training for the truck drivers who were involved in transporting the waste and fin ished products, there is little evidence of similar training among the operators who handled waste materials in the processing plant as part of their normal work. Only one site (11\%) had adequate arrangements for training.

\subsubsection{Risk and Incident Management}

The aim here was to assess whether hazards and system failures were reported, notified to government agencies where required, risk assessments conducted and documents maintained, methods used for risks were appropriate, work practices and protocols, documentation on instructions, risk assessments and safety data sheets, storage practices for hazardous substances and dangerous goods, labelling, and maintenance of plant, machinery and equipment. Emergency management policies and practices were also considered. These varied greatly at the facilities assessed. 67\% had systems and practice of notifying the relevant agency of any incidents, injuries and spills. Hazard, incident and system failures were reported in $56 \%$ of the workp laces. Effective methods of risk assessment, which took into account processes, existing controls, frequency of exposures and properties of chemical existed in 33\% of workplaces, while work practices and procedures for handling chemicals, scheduled and verifiable maintenance for plant, machinery and equipment existed in $44 \%$. The results in this study reflect also key areas of concerns identified the Superfund study[11], for example:

- absence of (formal or formal) health and safety plan (compared with hazard identification, risk assessment, procedures etc.), and

- engineering controls (compared maintenance of plant and systems of work).

The results are suggestive that employees working with the chemicals and hazardous wastes may not have complete access to information relating to safe use of chemicals (including material safety data sheets, documented risk assessments and work procedures for safe handling of chemicals). This may also explain why incompatible storage of chemicals were identified in over $50 \%$ of the workp laces; in the absence of appropriate procedures, most employees who handle or work with the chemicals may not be fully aware of the hazards and potential risks, and may not be able to apply principles of segregation, which is a higher-order engineering control than training.

Work procedures and practices, on their own, are rarely likely to prevent incidents and injuries. In fact, a planned approach to managing safety is crucial to the success of any program. A safety case, an environmental management plan, or a site specific health and safety plan provides a means to preventing chemical incidents. Key things to include in a site based health and safety plan include known measures that will prevent incidents and injuries. The USEPA for example, suggests, for example, the following to be included in a site health and safety plan:

- worker train ing;

- maintain ing mechanical integrity of process equipment;

- incident investigation and corrective actions;

- auditing; and

- substitution of hazardous chemicals with those that is less hazardous.

The preparedness of facilities in dealing with potential emergency situations through identification of threats and emergencies specific to the site's activities, establishment of written procedures to assist in response, allocation of responsibilities, and training of emp loyees and maintenance of fire protection equipment were also assessed. The results revealed that effective emergency management existed in only $11 \%$ of the facilities. Potential emergencies relevant to the site's operations had been identified and documented in $44 \%$, and site specific responsibilities were allocated in $78 \%$. However, employees had only been trained in $22 \%$ of the facilities, and $22 \%$ had an effective maintenance program for fire protection.

The results also showed that all nine facilities had a 'written' plan; however, these were very generic in nature, and had not been implemented or reviewed. Contact names and details were provided for a spectrum of control personnel. In one case, with four employees, the plan identified a Commander, Communications Officer, Area Warden and Deputy Warden. The responsibilities of each designation took up over $70 \%$ of the written plan. This is a reflection of 'shelved' emergency response plan that is common in many workp laces. The finding is very similar for that observed for health and safety polic ies, discussed earlier. It appears that writing up a policy and displaying it on the noticeboard in the workplace is in sync with emergency response plans developed by highly paid consultants and borrowed from the shelf. Both are likely to ineffective if 
users have not been trained in them.

The importance of a site specific emergency response and evacuation plan cannot be overemphasized, particularly if waste treatment facilities are located near other businesses. In essence, an emergency plan for a chemical waste treatment facility should be treated as a risk control measure, therefore needs to be developed following an assessment of all hazards, including that posed by fires and explosions.

\subsubsection{Monitoring and Review}

This section assessed the existence and effectiveness of any inspection or review plans, maintenance of engineering controls and general monitoring of employees and environment. The latter incorporated any internal and/or external audits undertaken by suppliers or government agencies or parent companies. $44 \%$ of the facilities had a scheduled maintenance program for engineering controls, $33 \%$ had program of monitoring the environ ment, emp loyees, visits by customers and government agencies. 22\% had a documented health and safety inspection program that was fully implemented, while $11 \%$ had fully implemented all elements of the monitoring and review.

The above results, when compared against the findings on management, planning and resources, suggests that access to safety expertise and/or resource could be contributing towards having systems which do not undergo any formal (or informal) review and auditing.

\section{Limitations and Conclusions}

In this project a multi-tiered approach was used to assess the health and safety performance across a sample of nine chemical waste treatment facilities in South-Eastern Victoria. Traditional safety performance indicators including injuries, claims and incidents data were first reviewed, followed by site inspection to identify workplace hazards. Auditing and verification of 28 sub-elements using SafetyMAP (4th Edition) were used to examine how well the facilities met compliance with health, safety, dangerous goods and major hazard facilities legislation. A total of 28 sub-elements grouped under management, resourcing and planning (5 sub-elements), consultation and employee engagement (5 sub-elements), employee training and development (3 sub-elements), risk and incident management (8 sub-elements), emergency management (4 sub-elements) and monitoring and review (3 sub-elements) were assessed. The results indicate that, generally: administrative arrangements, including policies and objectives, and procedures for dealing with issues exist in over $67 \%$ of the facilities; licenses, notifications and registrations meet legal requirements in $67 \%$ of the facilities. Responsibilities for emergency management existed in $78 \%$ of the facilities, and there is a wide variation in how well the facilities comply with other requirements. The results are particularly alarming for risk and incident management, and for emergency management, where $33 \%$ of facilities complied at best.

It is acknowledged the results are based on a small-sized non-random sample, and on a localised area of South Eastern Victoria, and based on convenience sampling. No statistical analysis has been performed to determine the significance of the results obtained. This pilot study did not examine the rehabilitation and management ofworkers who had been injured at work in these CWTFs. Moreover, workers exposure to occupational health and hygiene issues were also not considered. These are some areas that would need to be considered as part of a more comprehensive and in-depth study into the way health and safety and risks are managed in this sub-sector. As this study has demonstrated, safety auditing can be a useful tool for undertaking such assessments. It has been suggested that an expanded version of the tool developed here could also be us eful for examining aspects of safety culture[34-36], which has been suggested to be important for health and safety management, in CWTFs.

\section{ACKNOWLEDGEMENTS}

The author thanks the anonymous reviewers for their comments on this paper. The author also acknowledges the assistance of the nine organisations, the managers, supervisors, operators and health and safety representatives who freely gave their time and made available copies of documents to enable this pilot study to be conducted.

\section{REFERENCES}

[1] Australian Bureau of Statistics, "Waste Management Services, Australia 2009-10, ABS catalogue 8698.0," ABS, Ed., ed. Canberra: Australian Government Publishing Services, 2011.

[2] Occupational Health and Safety Act 2004. Victoria, Anstat Act No. 107/2004, 2004.

[3] Victorian WorkCover Authority, "Code of Practice No 27Storage and Handling of Dangerous Goods," V. W. Authority, Ed., ed. Melbourne, 2000.

[4] Victorian WorkCover Authority, "MHD GN-1. 2001. Notification and Registration under the Occupational Health and Safety (Major Hazard Facilities) Regulations 2000," M. H. Division, Ed., ed. Melbourne: Victorian WorkCover Authority, 2001.

[5] Victorian WorkCover Authority, "MHD GN-3. 2001. An Overview of the Safety Case Regime Under the Occupational Health and Safety (Major Hazard Facilities) Regulations 2000," M. H. Division, Ed., ed: Victorian WorkCover Authority, 2001.

[6] A. Robens, "Health and Safety at Work; Report of the Committee, 1970-1972," HMSO, London1972.

[7] V. Monk, "Occupational Health and Safety Management Systems and Safety Performance in the Building Industry: Report for the National Occupational Health and Safety 
Commission.," National Occupational Health and Safety Commission, Ed., ed. Sydney: Australian Government Publishing Service, 1995.

[8] O. M. Poulsen, N. O. Breum, N. Ebbehøj, Å. M. Hansen, U. I. Ivens, D. van Lelieveld, P. Malmros, L. Matthiasen, B. H. Nielsen, E. M. Nielsen, B. Schibye, T. Skov, E. I. Stenbaek, and K. C. Wilkins, "Sorting and Recycling of Domestic Waste. Review of Occupational Health Problems and Their Possible Causes," Science of The Total Environment, vol. 168, pp. 33-56, 1995.

[9] O. M. Poulsen, N. O. Breum, N. Ebbehøj, Å. M. Hansen, U. I. Ivens, D. van Lelieveld, P. Malmros, L. Matthiasen, B. H. Nielsen, E. M. Nielsen, B. Schibye, T. Skov, E. I. Stenbaek, and K. C. Wilkins, "Collection of Domestic Waste. Review of Occupational Health Problems and Their Possible Causes," The Science of the Total Environment, vol. 170, pp. 1-19, 1995.

[10] D. B. Riley and A. K. Weyman, "A Survey of Permit-to-work Systems and Forms in Small to Medium Sized Chemical Plant," presented at the ICHEME Sympodium Series No. 139, 1995.

[11] R. Fairfax, "Correcting Common Health and Safety Program Deficiencies at Remediation Sites," Applied Occupational and Environmental Hygiene, vol. ii, 1996.

[12] T. L. Gladding and P. C. Coggins, "Exposure to Microorganisms and Health Effects of Working in UK Materials Recovery facilities: a Preliminary Report," Ann Agric Environ Med, vol. 4, pp. 137-141, 1997.

[13] F. Lamm, "Small Business and OH\&S Advisors," Safety Science, vol. 25, pp. 153-161, 1997.

[14] D. McVittie, H. banikin, and W. Brocklebank, "The Effects of Firm Size on Injury Frequency in Constrcution," Safety Science, vol. 27, pp. 19-23, 1997.

[15] J. M. Eakin and E. MacEahen, "Health and the Social Relations of Work: A Study of the Health-Related Experiences of Employees in Small Workplaces," Sociology of Health and Illness, vol. 20, pp. 896-914, 1998.

[16] A. R. Hale, B. H. J. Heming, K. Smit, F. G. T. Rodenburg, and N. D. van Leeuwan, "Evaluating Safety in the Management of Maintenance Activities in the Chemical Process Industry," Safety Science, vol. 28, pp. 21-44, 1998.

[17] L. Vassie and S. Cox, "Small and Medium Size Enterprises (SME) Interest in Voluntary Certification Schemes for Health and Safety Management: Preliminary Results," Safety Science, vol. 29, pp. 67-73, 1998.

[18] H. An, J. Englehardt, L. Fleming, and J. Bean, "Occupational Health and Safety Amongst Municipal Solid Waste Workers in Florida " Waste Management \& Research, vol. 17, pp. 369-377, 1999.

[19] J. Harrison, J. Woodhouse, and A. J. Dowson, "The Management of Occupational Health by NHS Trusts in the North of England," Occupational Medicine, vol. 49, pp. 525-533, 1999

[20] L. Vassie, J. M. Tomàs, and A. Oliver, "Health and Safety Management in UK and Spanish SMEs: A Comparative Study," Safety Science, vol. 31, pp. 35-43, 2000.
[21] N. Bull, T. Riise, and B. E. Moen, "Work-related Injuries and Occupational Health and Safety factors in Smaller Enterprises - a Prospective Study," Occupational Medicine, vol. 52, pp. 70-74, 2002.

[22] D. Champoux and J.-P. Brun, "Occupational Health and Safety Management in Small Size Enterprises: an Overview of the Situation and Avenues for Intervention and Research," Safety Science, vol. 41, pp. 301-318, 2003.

[23] T. J. Larsson, "Is Small Business a Safety Problem?," Safety Science Monitor, vol. 7, 2003.

[24] M. Tischer and S. Scholaen, "Chemical Management and Control Strategies: Experiences From the GTZ Pilot Project on Chemical Safety in Indonesian Small and Medium-sized Enterprises," Annals of Occupational Hygiene, vol. 47, pp. 571-575, 2003

[25] D. Walters and F. Lamm. (2003, OHS in Small Organisations: Some Challenges and Ways Forward. Working paper 15, National Research Centre for OHS regulation, Australian National University.

[26] D. Walker and R. Tait, "Health and Safety Management in Small Enterprises: an Effective low cost Approach," Safety Science, vol. 42, pp. 69-83, 2004.

[27] M. Statheropoulos, A. Agapiou, and G. Pallis, "A Study of Volatile Organic Compounds Evolved in Urban Waste Disposal Bins," Atmospheric Environment, vol. 39, pp. 4639-4645, 2005.

[28] O. K. Tolvanen and K. I. Hänninen, "Occupational Hygiene in a Waste Incineration Plant," Waste Management, vol. 25, pp. 519-529, 2005.

[29] O. K. Tolvanen and K. I. Hänninen, "Mechanical-biological Waste Treatment and the Associated Occupational Hygiene in Finland," Waste Management, vol. 26, pp. 1119-1125, 2006.

[30] O. H. Sørensen, P. Hasle, and E. Bach, "Working in Small Enterprises - Is There a Special Risk?," Safety Science, vol. 45, pp. 1044-1059, 2007.

[31] N. K. Denzin and Y. S. Lincoln, Strategies of Qualitative Inquiry. Thousand Oaks: Sage, 1998.

[32] WorkSafe Victoria. (2002). SafetyMAP: Auditing Health and Safety Management (Fourth ed.). Available: http://www.safety risk.com.au/wp-content/uploads/download s/2010/06/SafetyM AP+auditing+management.pdf.

[33] Victorian WorkCover Authority Advisory Committee, "Incentives for Accident Prevention Among Small and Medium Sized Employers," V. W. Authority, Ed., ed. Melbourne, 1994.

[34] M. D. Cooper, "Towards a Model of Safety Culture," Safety Science, vol. 36, pp. 111-136, 2000.

[35] M. D. Cooper, Improving Safety Culture: a Practical Guide. Chichester: John Wiley \& Sons Ltd, 2001.

[36] M. D. Cooper, "Safety Culture," Professional Safety, vol. 47, 2002. 